\title{
The structure of frozen phases in slit nanopores: A grand canonical Monte Carlo study
}

\author{
K. G. Ayappa and Chandana Ghatak \\ Department of Chemical Engineering, Indian Institute of Science, Bangalore, India 560012
}

\begin{abstract}
Freezing of soft spheres in slit nanopores is investigated using Grand canonical Monte Carlo simulations. The pores are in equilibrium with a liquid located close to the liquid-solid coexistence region in the bulk Lennard-Jones phase diagram. In addition to layering, the confined fluid is found to possess in-plane order, leading to the formation of frozen phases which give rise to a sequence of solid-solid transformations as the pore width is varied. Transformations between $n$ layered triangular to $n+1$ layered square lattices and between $n$ layered square to triangular lattices, are observed for $n=1,2,3$, and 4 . The transition from triangular to square lattices occurs via an intermediate buckled phase which is characterized by increased out-of-plane motion, while maintaining in-plane triangular order. Buckling was found to decrease with increasing number of layers. The transition between square to triangular lattices at a fixed number of layers is accompanied by a lowering of the solvation force, resulting in a doublet in the solvation force maxima. Influence of fluid-wall interactions on the nature of the frozen phases are studied by comparing the structures formed with a 10-4-3 and 10-4 fluid-wall potential. The solid structures are classified based on their closest 3D counterparts.
\end{abstract}

\section{INTRODUCTION}

The understanding of the structure and dynamics of molecularly thin confined fluids is important in processes such as wetting, adhesion, coatings, and boundary lubrication. Although the gas to liquid transition in micropores is reasonably well understood, the liquid to solid transition and the nature of possible solid phases that can exist under confinement has only recently received greater attention.

Perhaps the most widely used experimental tool for probing the physics of molecularly confined fluids is the surface force apparatus (SFA), where forces across a fluid confined between two mica (atomically smooth) cross cylinders are measured. ${ }^{1}$ Although primarily developed to measure forces across molecularly thin films, dynamic SFA experiments where the confined fluid is subject to normal and tangential forces can be used to indirectly infer the state of the confined fluid. Dynamic SFA experiments with nonpolar organic molecules, such as cyclohexane, indicate the presence of a liquid to solid transition solely due to increasing confinement. ${ }^{2}$ Solidification is inferred by the ability of the film to support a finite shear stress. Dynamic stick-slip during sliding experiments has also been attributed to alternate freezing and melting mechanisms. ${ }^{3}$ Fundamentally, solidification would imply that in addition to layering (which is well established) the fluid would possess long range in-plane order as well. Since SFA experiments cannot establish this directly, the exact nature of the "solid" phase and the liquidsolid transition is still under debate, with the possibility of the formation of an intermediate amorphous glassy state. ${ }^{4,5}$ Recent extensions of the SFA (Ref. 6) indicate that a combination of the SFA with improved optical imaging techniques have the potential to yield a more complete physiochemical picture of the confined fluid.

Molecular simulation techniques have provided a powerful means of interpreting experimental data and have aided in developing theories for inhomogeneous fluids. It is well established, both by computer simulation ${ }^{7-9}$ and density functional theory, ${ }^{10}$ that the oscillatory solvation force as a function of confinement is accompanied by the formation and disruption of layers. Molecular simulations that most appropriately describe the conditions in a typical SFA experiment are the grand canonical Monte Carlo (GCMC) simulations. In a GCMC simulation a pore of given volume $V$ is equilibrated with a bulk fluid of chemical potential $\mu$ and temperature $T$. In molecular simulations the pore walls are modeled as either structured or smooth. In a structured pore the fluid-wall potential is dependent on the lattice positions of atoms that make up the wall. In the smooth wall the fluidwall potential is only a function of the normal distance of the fluid atom from the wall. Epitaxial freezing occurs in structured, commensurate pores. ${ }^{8,11,12}$ GCMC simulations have shown that depending on the bulk chemical potential, the pore fluid need not always epitaxially freeze in structured pores. ${ }^{13}$ On the other hand, under suitable thermodynamic conditions, freezing can occur in pores with atomically smooth surfaces ${ }^{14-16}$ and in structured pores when the adsorbed fluid is incommensurate with the pore walls, ${ }^{17}$ indicating that epitaxy is not a necessary condition for freezing of the confined fluid. ${ }^{2}$

With the development of experimental techniques, the nature of frozen phases, with emphasis on the liquid to solid transition under confinement, have received greater attention. ${ }^{18}$ The underlying inhomogeneous structure of the confined fluid and nature of the pore-fluid interaction can 
either elevate or depress the freezing point relative to the bulk fluid. ${ }^{19-21}$ Studies which investigate the structure of frozen water under confinement, indicate that the structures and transitions can be quite different from the bulk counterparts. $^{22,23}$ Molecular dynamics simulations of water in a slit pore of $1 \mathrm{~nm}$ width predicts the formation bilayer ice crystals, with slightly distorted hexagonal rings. ${ }^{24}$ Molecular simulations of nitrogen in slit graphite pores show that ordered phases that are not observed during adsorption on a single graphite sheet can be stabilized under confinement. ${ }^{25}$ $\mathrm{X}$-ray diffraction studies of $\mathrm{Ar}$ and $\mathrm{Kr}$ in Vycor glass reveal the presence of randomly stacked layers giving rise to a disordered hexagonal close packed structure, and the presence of a solid-solid transition between this phase and the face centered cubic structure. ${ }^{26}$ More recently, x-ray diffraction studies have been reported for the liquid-solid and solidsolid transitions for oxygen in cylindrical pores ${ }^{27}$ and melting and freezing of argon in porous silica. ${ }^{28}$

Unlike molecularly confined films, colloidal suspensions confined between glass plates can be directly imaged using optical microscopy techniques. As a result solid-solid transitions in high density colloidal suspensions have been studied using a combination of experiments ${ }^{29-31}$ and computer simulations. ${ }^{32-35}$ The confined colloidal suspension has provided a versatile experimental system to explore the liquidhexatic-solid phase transitions in quasi-two-dimensional systems. ${ }^{36,37}$ In the single layer regime the solid freezes into a triangular lattice and when more than one layer is present, square lattices are formed at low densities, which transform into triangular lattices at higher densities. The transition from $n$ layered triangular to $n+1$ layered square lattice is seen to proceed through buckled and prism phases. ${ }^{38}$ This sequence of transitions, in these reduced dimensional systems, from square to triangular lattices is understood predominantly in terms of excluded volume effects. ${ }^{39}$

We have recently carried out GCMC simulations for a Lennard-Jones (LJ) fluid confined to a smooth slit graphite pore. ${ }^{16}$ Unlike previous GCMC studies in slit pores, we considered a high density bulk fluid located close to the liquidsolid freezing line. Under these conditions our preliminary investigation reveals that in addition to the fluid freezing in the pore, solid-solid transitions between triangular and square lattices, which are remarkably similar to the sequence observed in colloidal suspensions are observed. Since excluded volume effects dominate the structure of high density soft-sphere systems this qualitative similarity between micron sized colloidal suspensions and molecular thin films is not completely unexpected. An earlier GCMC study ${ }^{17}$ which reveals the presence of a solid-solid transition in a soft sphere LJ system showed that a transition from square (bcc) to triangular (triclinic) lattices in the bilayer regime for xenon confined in a structured pore-wall made up of argon atoms. In the same study solid-solid transitions were not observed for the commensurate system formed by replacing xenon with argon. Perhaps the lower bulk fluid density used in these simulations, restricted the transformations to the bilayer regime.

In this manuscript, we extend our preliminary communication ${ }^{16}$ and present a more detailed analysis of the struc- tures of frozen phases in slit shaped pores. GCMC simulations are carried out for the similar bulk state point used earlier. ${ }^{16}$ Here, the structure of the confined phases are contrasted for two kinds of smooth pore systems. The first is one is methane adsorbed in graphite, which represents a system with strong fluid-wall interactions. The second is a pore modeled using the 10-4 fluid-wall potential with argon as the confined fluid. When compared with the 10-4-3 fluidwall potential, the 10-4 potential represents a weakly attractive pore. The confined fluid structures are examined by computing in-plane pair correlation functions, in-plane bond order parameters, density distributions, and snapshots. The frozen phases are classified based on their three dimensional counterparts and where possible we compare our observations with the crystal structures observed in hard sphere and confined colloidal systems.

\section{THEORY AND SIMULATION PROCEDURE}

\section{A. Fluid-fluid potentials}

Fluid-fluid interactions are modeled using a 12-6 LJ potential,

$$
u\left(r_{i j}\right)=4 \epsilon_{f f}\left[\left(\frac{\sigma_{f f}}{r_{i j}}\right)^{12}-\left(\frac{\sigma_{f f}}{r_{i j}}\right)^{6}\right],
$$

where $\epsilon_{f f}$ and $\sigma_{f f}$ are the LJ energy and size parameters, respectively, and $r_{i j}$ is the distance between particles $i$ and $j$.

\section{B. Fluid-wall potentials}

In order to study the influence of the wall potential on the structure of the confined fluid, two smooth wall systems have been investigated. In the first case the fluid-wall interaction potential is modeled using a $10-4-3$ potential, ${ }^{40}$

$$
\begin{aligned}
U_{f w}(z)= & 2 \pi \rho_{w} \epsilon_{f w} \sigma_{f w}^{2} \Delta\left[\frac{2}{5}\left(\frac{\sigma_{f w}}{z}\right)^{10}-\left(\frac{\sigma_{f w}}{z}\right)^{4}\right. \\
& \left.-\frac{\sigma_{f w}^{4}}{3 \Delta(z+0.61 \Delta)^{3}}\right]
\end{aligned}
$$

where $z$ is the perpendicular distance between the fluid particle and the wall, $\rho_{w}$ is the surface density of wall atoms, $\Delta$ is the interlayer spacing of wall atoms, $\epsilon_{f w}$ is the fluid-wall interaction parameter, and $\sigma_{f w}$ is the fluid-wall diameter. Earlier studies for freezing in this system have shown that the structure of the frozen phase is not influenced by a more detailed fluid-wall potential describing the corrugation of the graphite sheet. ${ }^{15}$ The pore is assumed to be periodic in the $x-y$ plane.

In the second system studied the fluid-wall potential is a 10-4 potential,

$$
U_{w f}(z)=2 \pi \epsilon_{f w}\left[\frac{2}{5}\left(\frac{\sigma_{f w}}{z}\right)^{10}-\left(\frac{\sigma_{f w}}{z}\right)^{4}\right] .
$$

Unlike the 10-4-3 potential which takes into account the multilayered nature of the wall atoms, the 10-4 potential in Eq. (3) represents the interaction between the fluid atom and a single plane (111) of a fcc lattice. The complete fluid-wall 
TABLE I. Fluid-fluid and fluid-wall potential parameters used in this study.

\begin{tabular}{lcccc}
\hline \hline Parameter & Methane $^{\mathrm{a}}$ & Argon & Wall 10-4-3 & Wall 10-4 \\
\hline$\epsilon_{f f}(\mathrm{~K})$ & 148.1 & 120 & $\ldots$ & $\ldots$ \\
$\epsilon_{w w}(\mathrm{~K})$ & & & 28.0 & 120 \\
$\sigma_{f f}(\AA)$ & 3.8 & 3.405 & $\ldots$ & $\ldots$ \\
$\sigma_{w w}(\AA)$ & & & 3.40 & 3.405 \\
$\rho_{w}\left(\AA^{-3}\right)$ & & 0.114 & \\
$\Delta(\AA)$ & & 3.35 & \\
\hline \hline
\end{tabular}

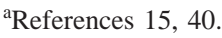

interaction potential from two walls for a pore of width $H$, one located at $z=-H / 2$ and the other at $z=H / 2$ is

$$
V_{f w}=U_{f w}(z+H / 2)+U_{f w}(H / 2-z) .
$$

The fluid and wall interaction parameters used in the simulations are indicated in Table I. For the 10-4-3 wall potential the interaction parameters are similar to those used for methane on graphite ${ }^{15}$ and for the 10-4 wall, the parameters are those of argon. The fluid wall interaction parameters were computed using the standard Lorentz-Berthelot mixture rules,

$$
\sigma_{f w}=\frac{\sigma_{f f}+\sigma_{w w}}{2} \text { and } \epsilon_{f w}=\sqrt{\epsilon_{f} \epsilon_{w}} .
$$

\section{Layer density distributions}

The layer density distribution,

$$
\rho(z)=\frac{\left\langle N\left(z-\frac{\Delta z}{2}, z+\frac{\Delta z}{2}\right)\right\rangle}{A \Delta z},
$$

where $\langle N(z-(\Delta z / 2), z+(\Delta z / 2))\rangle$ is the ensemble averaged number of atoms in a bin of thickness $\Delta z$ in the $z$ direction, $A=L^{2}$ is the area of the fluid layer for the simulation box of length $L$ in the $x-y$ plane. A bin width $\Delta z=0.025 \sigma$ was used in the simulation.

\section{Solvation force}

The solvation force for the fluid confined in a slit pore of area $A$ is defined as

$f_{z}=-\frac{1}{2 A} \sum_{i=1}^{N}\left\langle\frac{d U_{f w}\left(z_{i}+H / 2\right)}{d z_{i}}+\frac{d U_{f w}\left(H / 2-z_{i}\right)}{d z_{i}}\right\rangle$,

where $z_{i}$ is the position of fluid particle $i$. The solvation force is a sum of the contributions of the force exerted by the fluid

TABLE II. Reduced units used in this work. $\epsilon_{f f}$ and $\sigma_{f f}$ are the LennardJones parameters for the fluid-fluid interactions.

\begin{tabular}{ll}
\hline \hline Quantity & Reduced unit \\
\hline Solvation force & $f_{z}^{*}=f_{z} \sigma_{f f}^{3} / \epsilon_{f f}$ \\
Pore width & $H^{*}=H / \sigma_{f f}$ \\
Pressure & $P^{*}=P \sigma_{f f}^{3} / \epsilon_{f f}$ \\
Temperature & $T^{*}=k T / \epsilon_{f f}$ \\
Density & $\rho^{*}=\rho \sigma_{f f}^{3}$ \\
Activity & $Z^{*}=Z \sigma_{f f}^{3}$ \\
\hline \hline
\end{tabular}

molecules on both the confining walls. The disjoining pressure is defined as the difference between the solvation force and the bulk pressure of the fluid which is in equilibrium with the pore. In an open system, where the pore is in equilibrium with a bulk fluid, the disjoining pressure approaches zero for large pore widths.

\section{E. Bond angle order parameters}

The local bond angle order parameters,

$$
\psi_{n}=\left\langle\frac{1}{N_{b}}\right| \sum_{j=1}^{N_{b}} \exp \left(\text { in } \theta_{j}\right)|\rangle,
$$

where $\theta_{j}$ is the bond angle formed between an atom and its nearest neighbors $\left(N_{b}\right)$ with reference to a fixed reference frame, are computed for $n=4,6$. A distance cutoff of $1.25 \sigma_{f f}$ was used while computing the nearest neighbor bond distances for the bond order parameters. The bond order parameters which are computed for individual layers (averaging over atoms) are averaged over the different layers in the pore. The value of $\psi_{n}$ varies between 0 and $1 . \psi_{4}=1$, corresponds to a perfectly square lattice and $\psi_{6}=1$ indicates a triangular lattice. Deviations of the value of $\psi_{n}$ from unity indicate the degree of disorder in the lattice.

\section{F. Pair correlation function}

The pair correlation function (PCF) is computed within individual layers using

$$
g(r)=\left\langle\frac{N(r, \Delta r ; \Delta z)}{N_{l} \rho_{l}(z) 2 \pi r \Delta r \Delta z}\right\rangle,
$$

where, $\langle N(r, \Delta r ; \Delta z)\rangle$ is the number of fluid atoms in a cylindrical shell of radius $r$, thickness $\Delta r$, and width $\Delta z \cdot \rho_{l}(z)$ is the layer density in a bin of volume $A \Delta z$ and $N_{l}$ is the number of atoms in the layer.

\section{G. Bulk fluid and pore GCMC}

All results are reported in reduced units as shown in Table II. Prior to carrying out the pore fluid studies GCMC simulations were carried out to establish the bulk thermodynamic state. Using a cubic simulation box of length of $7 \sigma$, potential cutoff of $3.5 \sigma$ with long range corrections, the density corresponding to an activity, $Z^{*}=Z \sigma_{f f}^{3}=1.2, T^{*}$ $=k T / \epsilon_{f f}=1.0$, where $k$ is the Boltzmann constant is $\rho^{*}$ $=0.898$. The location of this point is illustrated in the bulk LJ phase diagram ${ }^{41,42}$ shown in Fig. 1, where the location of the liquid state points used in some of the earlier GCMC simulations ${ }^{8,9,43}$ are shown for comparison. As an independent check we used the 32 parameter LJ equation of state, ${ }^{44}$ which predicted a bulk density of 0.9001 and corresponding pressure $P_{b}^{*}=3.32$ at $Z^{*}=1.2$.

For the pore GCMC $(\mu V T)$ simulations, $4-5 \times 10^{6}$ Monte Carlo moves were used for equilibration, followed by $5-8 \times 10^{6}$ moves during which ensemble averages were collected. Each Monte Carlo move consists of an attempted addition, deletion and displacement with equal probability. ${ }^{45}$ Longer runs especially in the regions where the phase transitions occurred did not alter the results. A square simulation 

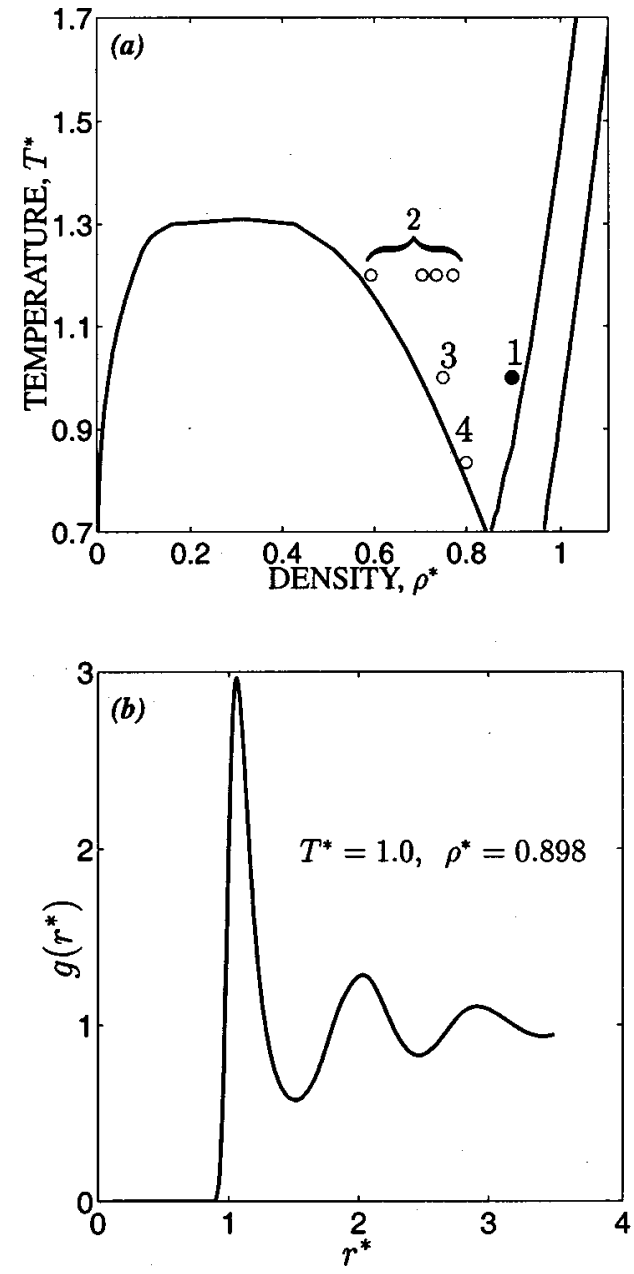

FIG. 1. (a) Location of the state point used for GCMC simulations illustrated on the bulk LJ phase diagram. The liquid-vapor coexistence data are from Lofti et al. (Ref. 42), and the liquid-solid coexistence data are from Agarwal and Kofke (Ref. 41). Note the proximity of the state point, " 1 ", used in this study, $\left(T^{*}=1.0, \rho^{*}=0.898\right)$ to the liquid-solid transition line. State points, "2" (Ref. 43), "3" (Ref. 8), "4" (Ref. 9) used in earlier GCMC simulations are indicated for comparison. (b) Pair correlation function for state point " 1 " illustrated in (b).

box was employed in all cases with periodic boundary conditions in the $x-y$ plane. Systematic system size checks were carried out with two simulation box lengths, $L=7 \sigma_{f f}$ and $L=9 \sigma_{f f}$ and in a few cases $L=12 \sigma_{f f}$. Small differences were observed in the pore densities between the $L=7 \sigma_{f f}$ and $9 \sigma_{f f}$, with greatest variations for pore widths at which solid-solid transitions occurred. However the pore widths at which the transitions occurred were unaltered. The results reported here correspond to simulations carried out with a periodic box of length $9 \sigma_{f f}$ and a potential cutoff of $4.5 \sigma_{f f}$. In the single layer regime, a box length of $12 \sigma_{f f}$ was used. Figure 2 illustrates the density distributions obtained for a 10-4 pore of width $H^{*}=16 \sigma_{f f}$, where the central fluid layers achieve the density of the bulk fluid. The corresponding solvation force at this pore width, $f_{z}^{*}=3.28$, is in good agreement with the pressure of the bulk fluid. The pore density is evaluated using, $\rho_{p}=\langle N\rangle / V_{p}$, where $\langle N\rangle$ is the ensemble averaged number obtained in the GCMC simulation and the pore volume $V_{p}=L^{2} H$.

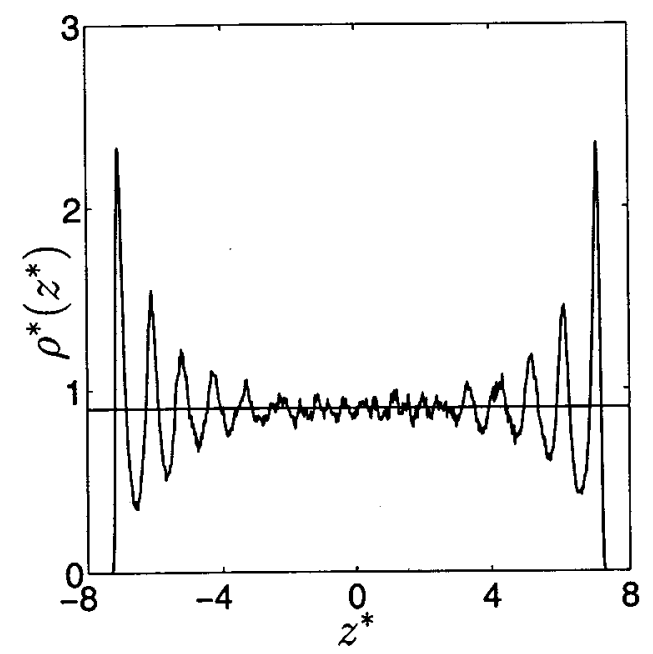

FIG. 2. Density distribution for a 10-4 fluid-wall potential [Eq. (11)] pore with $2 \pi \epsilon_{f w}^{*}=1$ and $H^{*}=16 \sigma_{f f}$. For reference, the density of the bulk fluid, $\rho^{*}=0.898$ is indicated as a horizontal line. At this pore width, the solvation force $f_{z}^{*}=3.28$.

\section{RESULTS}

The results for the 10-4-3 pore will be discussed first, by examining the structure of the confined layers in the different regimes as revealed by the density distributions, PCF's, snapshots, solvation force curves and order parameters. This is followed by a comparison with the results obtained for the 10-4 pore.

\section{A. Single layer}

The density distributions for the single layered regime are shown in Fig. 3(a), with PCF's and side view snapshots shown in Figs. 3(b) and 3(c), respectively. From the PCF's and the values of $\psi_{6}$ it is clear that the atoms adopt a triangular lattice structure. The vertical dashed lines in Fig. 3(b) correspond to the lattice positions of an ideal $111 \mathrm{fcc}$ lattice, based on the lattice parameter obtained from the first peak of the PCF. For pore widths $H^{*} \leqslant 1.9$, the density distributions [Fig. 3(a)] and the side view snapshots [Fig. 3(c)] indicate that the atoms occupy the central region of the pore with little out-of-plane movement. For $2.0 \leqslant H^{*} \leqslant 2.25$ the density distributions broaden and increased out-of-plane motion is observed [Fig. 3(c)]. However the order parameters and PCF's indicate that a triangular lattice is still retained, giving rise to the buckled phase. The buckled phase occurs when particles are able to move out-of-plane in the $z$ direction, while maintaining in-plane order. This situation occurs for $2.0 \leqslant H^{*} \leqslant 2.25$.

Snapshots of the $x-y$ plane shown in Figs. 4(a) and 4(b) for $H^{*}=1.82$ and 2.25, respectively, reveal the formation of a triangular lattice where each atom is surrounded by six nearest neighbors. The lattice is however not free of defects. The presence of defects is best revealed by the corresponding Voronoi polyhedra which is equivalent to the Wigner Seitz cells. The Voronoi poylyhedra around each atom is the polygon formed with the perpendicular bisectors of the lines joining the central atom with its nearest neighbors. In a triangu- 

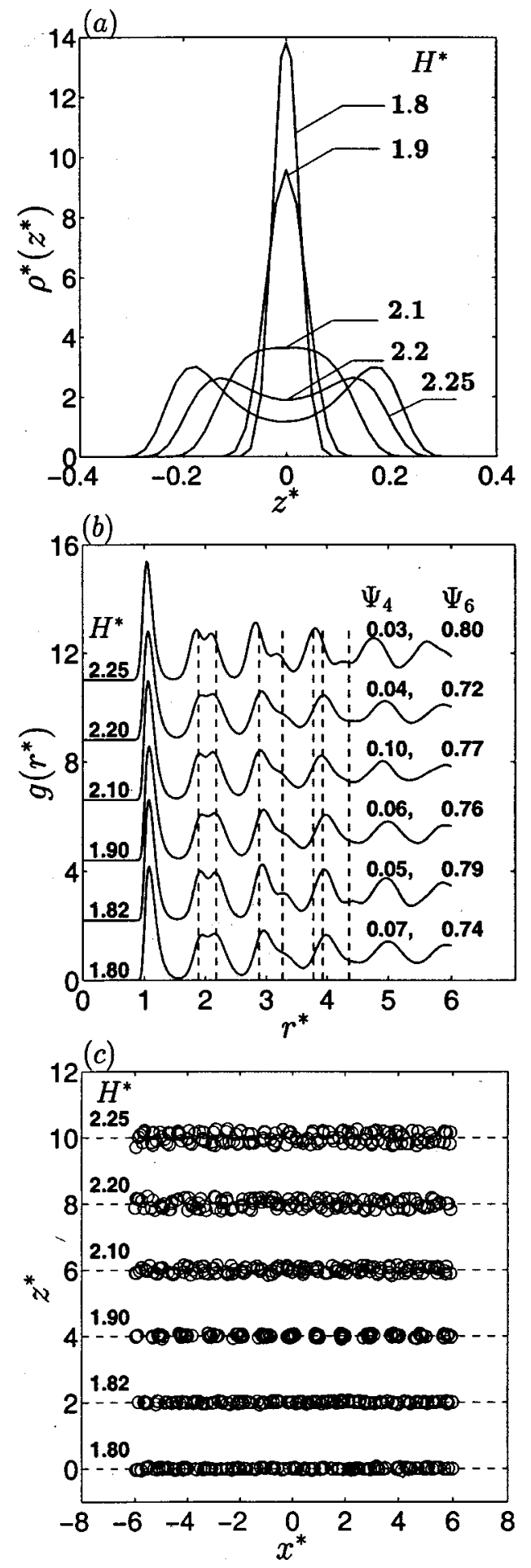

FIG. 3. (a) Density distributions, (b) pair correlation functions, and (c) side view snapshots for the single layer regime; $10-4-3$ fluid-wall potential. The pair correlation functions indicate the presence of a triangular lattice structure. For $H^{*}>2.1$, out-of-plane motions give rise to a buckled lattice. At $H^{*}=2.25$ where buckling is the greatest, as observed in the side view snapshots, the pair correlation functions indicate that the in-plane triangular lattice is retained.

lar lattice, polygons with the number of sides differing from six, indicate the presence of defects. The Vornoi diagram at $H^{*}=2.25$ clearly indicate the presence of a pair of pentagons in the region of the defect, marked with the letter $\mathbf{D}$.
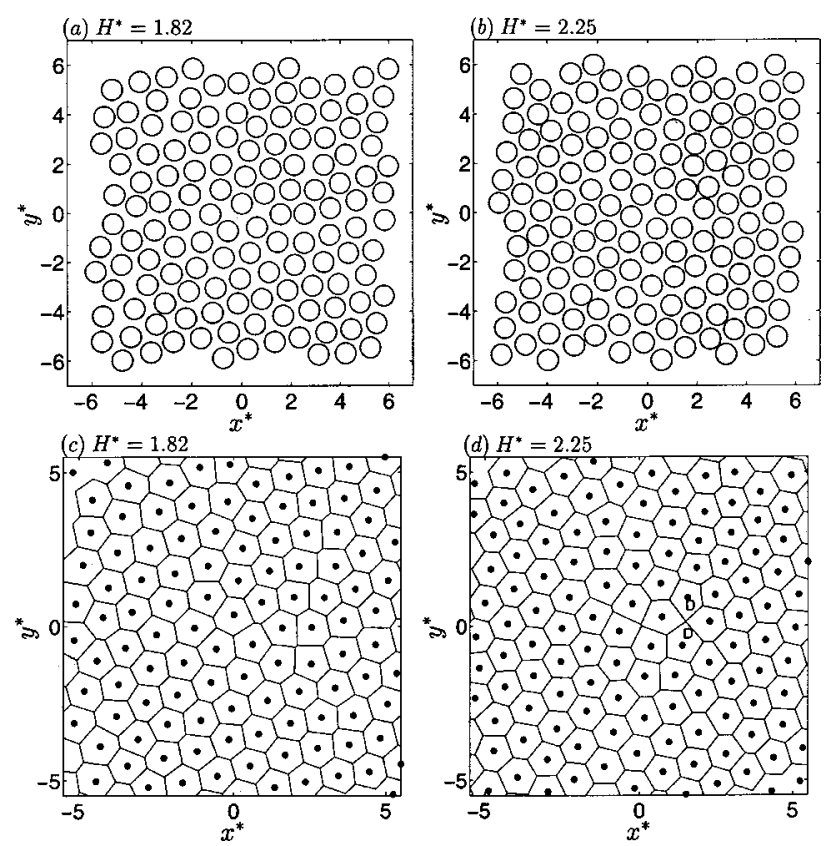

FIG. 4. Snapshots and corresponding Voronoi diagrams for $H^{*}=1.82$ and 2.25. For $H^{*}=2.25$, where buckling is significant as seen in Fig. 3(c), the atoms shift out-of-plane in a random manner with no specific pattern, giving rise to random buckling. The pairs of five sided polygons in the Voronoi diagrams indicate the presence of defects in the triangular lattices. These defects have been marked with the letter "D" in (d).

\section{B. Two layer}

As the pore width is increased a transition from one to two layers occurs with the first two layers appearing at $H^{*}$ $=2.39$ as seen in the density distributions [Fig. 5(a)]. Since the PCF's in both layers were identical, the PCF's for only one of the wall layers are shown in Fig. 5(b). The square lattice starts to form at $H^{*}=2.39\left(\psi_{4}=0.84\right)$ where the peaks in the PCF are broadened. At $H^{*}=2.45\left(\psi_{4}=0.93\right)$ the peaks in the PCF are well defined, and similar in intensity and location to the peaks shown for $H^{*}=2.48$ in Fig. 5 (b). Beyond $H^{*}=2.45$ the peaks clearly reveal the presence of a square lattice and the order parameter $\psi_{4}$ decreases continually until $H^{*}=2.58$. For $2.58<H^{*} \leqslant 2.66$, in-plane order once again increases and the fluid transforms to a triangular (111 fcc) lattice for $2.66<H^{*}<2.68$ as seen in the corresponding PCF [Fig. 5(b)]. The triangular lattice structure persists until $H^{*}=3.1\left(\psi_{6}=0.73\right)$, beyond which the fluid enters into a narrow transition regime between two and three layers as indicated by the density distributions at $H^{*}=3.2$ [Fig. 5(a)]. Similar to the single layer situation, a buckled triangular phase is observed for $2.9 \leqslant H^{*} \leqslant 3.1$. The side view snapshots [Fig. 5(c)] indicate the relative increase in buckling as the pore width is increased.

Snapshots of the atomic position shown in Fig. 6(a) reveal the formation of the square lattice at $H^{*}=2.48$. The lattice structure at $H^{*}=2.6$ which corresponds to a pore width at which the transition from a square to triangular structure [Fig. 6(b)] occurs, reveals the formation of a triangular lattice as shown in the upper right-hand side of the snapshot [Fig. 6(b)] which coexists with the predominantly square lattice domains in the lower left corner. At $H^{*}$ 

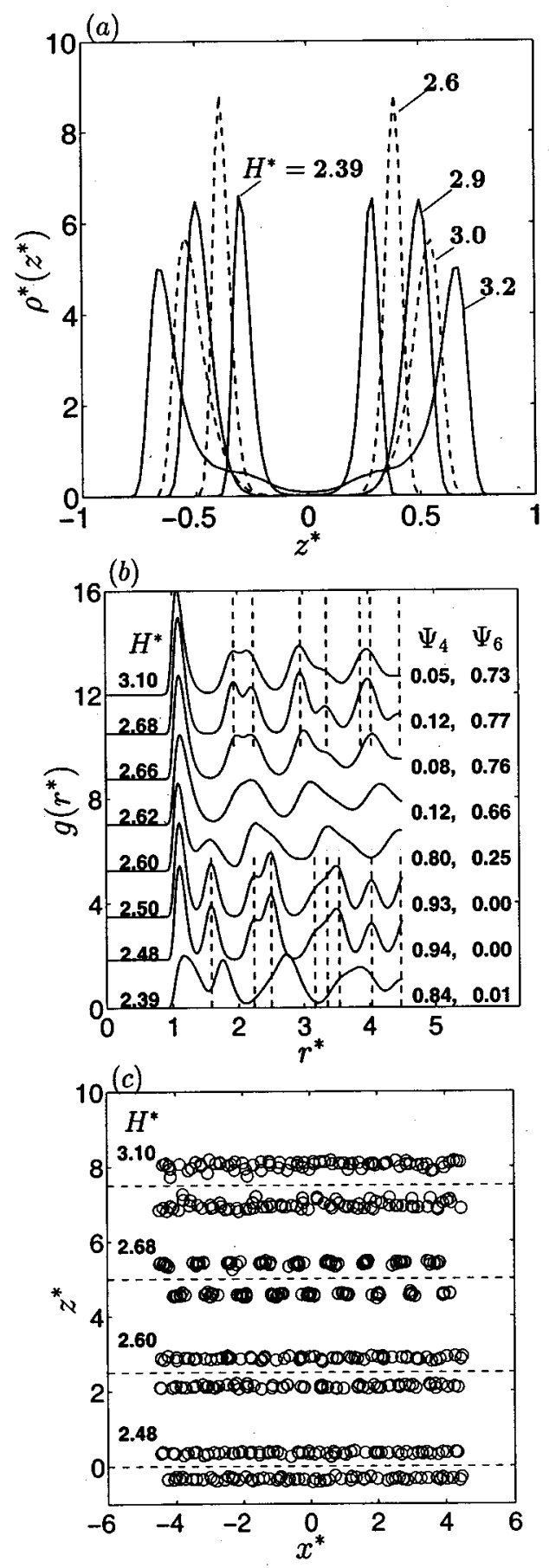

FIG. 5. (a) Density distributions, (b) pair correlation functions, and (c) side view snapshots for the two layer regime; 10-4-3 fluid-wall potential. The two layered regime exists for $2.39 \leqslant H^{*} \leqslant 3.1$. Buckling occurs for 2.9 $\leqslant H^{*} \leqslant 3.1$ beyond which the fluid enters into a transition regime between two and three layers.

$=2.68[$ Fig. $6(\mathrm{c})]$ the snapshots reveal the formation of a triangular lattice, with the atoms in each layer located in the three fold sites created by the adjacent layer. At $H^{*}=3.1$ the snapshots indicate some interesting structural details. The atoms in the top layer (shaded circles) no longer occupy the three fold sites formed by the bottom fcc lattice [as seen in Fig. 6(c)] but are shifted preferentially toward one of the three underlying atoms. This occurs for $2.9 \leqslant H^{*} \leqslant 3.1$ indicating that the relative shifting of atoms from their ideal
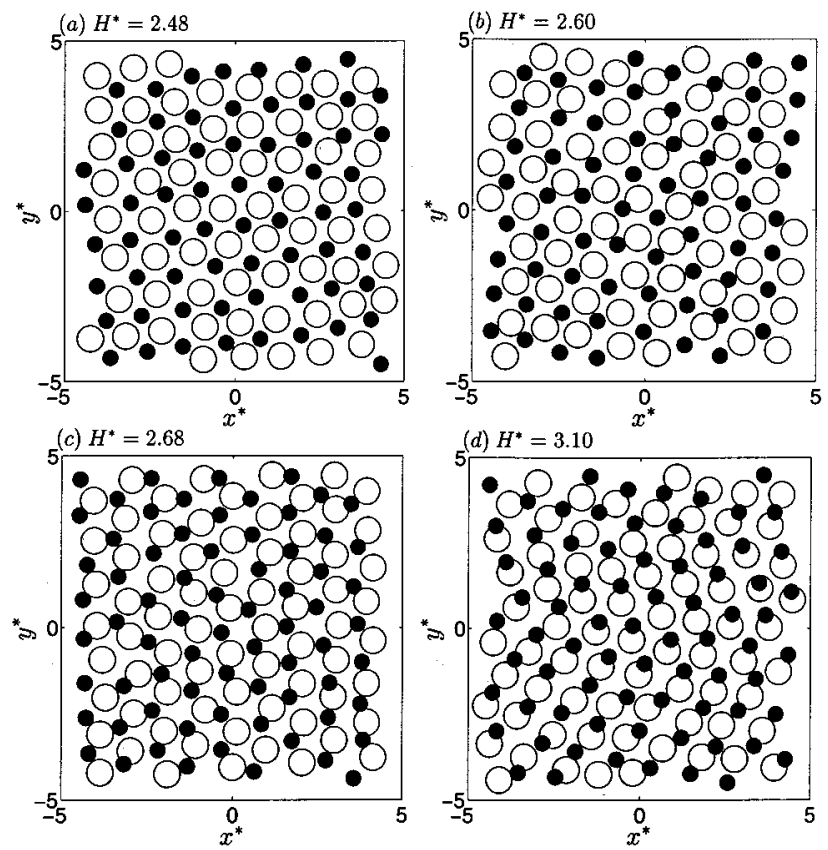

FIG. 6. Snapshots in the two layered regime indicate the transition from square lattices, at (a) $H^{*}=2.48$ to triangular lattices (c) $H^{*}=2.68$. At $H^{*}$ $=2.60$ (b) where the transition occurs the lattice consists of predominantly square lattices with smaller regions of triangular lattices (upper right-hand corner). At $H^{*}=3.1$ (d) although the triangular lattice is preserved in both layers the top layer (shaded circles) no longer occupy the threefold sites created by the bottom layer (open circles) as seen at $H^{*}=2.68$ (c).

locations occurs with increasing interlayer distance. Within each layer, however, the triangular lattice is retained as seen in the PCF for $H^{*}=3.1$ [Fig. 5(b)].

\section{Three and four layers}

In the three layered situations, the structures are able to form a complete unit cell in the direction of confinement. The corresponding PCF plots for the three layered system are shown in Fig. 7(a). The PCF's for the contact (adjacent to the wall) layer and middle layer are shown for $3.3 \leqslant H^{*} \leqslant 4.0$. The presence of order in the contact layers is seen to induce greater order in the middle layer indicating that the contact layer plays the role of a psuedostructured wall, into which the middle layer "epitaxially" orders. This increased ordering, in the inner layers was clearly perceptible only for the square lattices. The square lattice exists for $3.3 \leqslant H^{*} \leqslant 3.5$. At $3.5<H^{*}<3.55$ where the transition from a square to triangular lattice occurs, the PCF's indicate that disorder first occurs in the wall layer. At $H^{*}=3.6$ the fluid transforms into a triangular lattice.

In addition to the square to triangular lattice transition, an additional phase was observed in the three layered triangular lattice based on the stacking of individual layers. Examination of the stacking sequence in the three layered lattices reveals an ABA stacking for $3.6 \leqslant H^{*}<3.8$ corresponding to the hcp lattice. The snapshot in Fig. 8(b) reveals this structure at $H^{*}=3.7$. At $H^{*}=3.8$ the stacking sequence transforms to the ABC stacking of a fcc crystal. For 3.8 $<H^{*} \leqslant 4.1$ although the in-plane triangular lattice structure is preserved, the relative arrangement of atoms between 

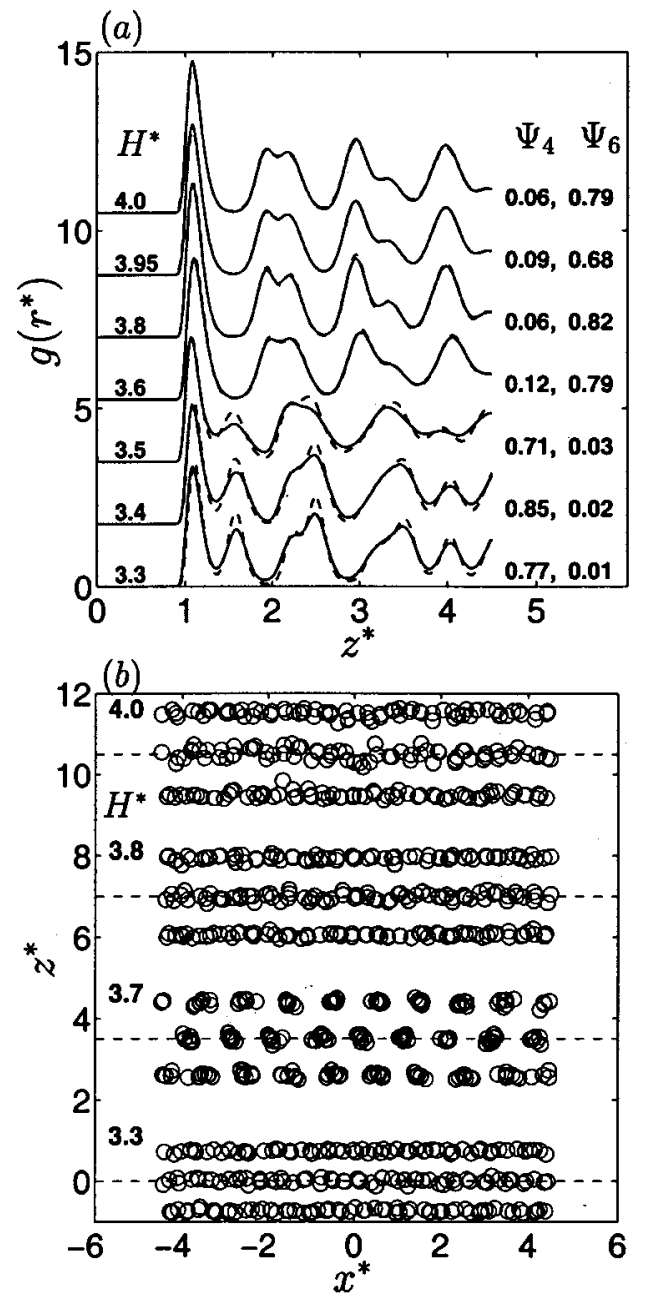

FIG. 7. (a) Pair correlation functions and (b) side view snapshots in the three layered regime, $3.3 \leqslant H^{*} \leqslant 4.1 ; 10-4-3$ fluid-wall potential. Buckling occurs for $H^{*}>3.8$, with greater out-of-plane movement observed in the central layers. The central layer is more structured than the wall layer when square lattices form, $3.3 \leqslant H^{*} \leqslant 3.5$, although no such increased order was observed with triangular lattices.

layers deviates from the ideal lattice structures. Out of plane displacements (buckling) in the central layer for $H^{*}>3.8$ increase sharply [Fig. 7(b)] with deviations as large as $0.5 \sigma_{f f}$ at $H^{*}=4.0$. The in-plane triangular structure persists until $H^{*}=4.1$ above which the transition into four layers occurs.

Figure 9 illustrates the density distributions, PCF's, and selected snapshots in the four layered regime. Four layers are observed at $H^{*}=4.2$ [Fig. 9(a)] and both the contact and inner layers are disordered as seen in Fig. 9(b). At $H^{*}$ $=4.3$, where the layers are well defined, the atoms form a square lattice and at $H^{*}=4.5$, a triangular lattice is observed. The stacking for the square and triangular lattices is $\mathrm{ABAB}$ as seen in Figs. 9(c) and 9(d), respectively. We did not carry out simulations beyond $H^{*}=4.5$.

\section{Solvation force and order parameters}

Figure 10(a) shows the solvation force and bond angle order parameter $\psi_{4}$ as a function of the reduced pore width $H^{*}$ and both the order parameters $\psi_{4}$ and $\psi_{6}$ are compared in Fig. 10(b). Typical error bars associated with the solvation
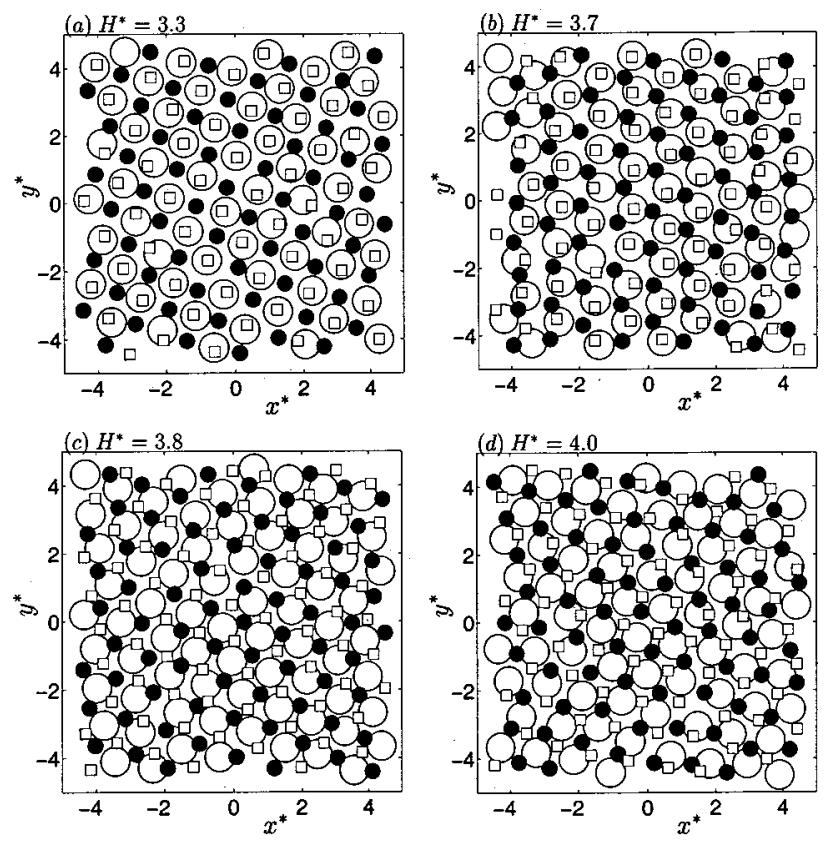

FIG. 8. Top view snapshots in the three layered regime. Open circles, bottom layer $(z \leqslant 0)$; filled circles, middle layer; open squares, top layer. The square lattice exists at $H^{*}=3.3$ (a) where the atoms on each layer occupies the fourfold site created by the bottom layer. A triangular lattice with ABA stacking is observed at $H^{*}=3.7$ (b) which transforms to ABC stacking at $H^{*}=3.8$. At $H^{*}=4.0$ the structures are buckled [Fig. 7(b)] however, the in-plane triangular lattice is preserved. The shifting away from the threefold sites as observed in the two layer [Fig. 6(d)] situation is observed here as well.

force data reported here are illustrated in Fig. 11. The maxima in the solvation force correspond to well ordered fluid layers and the minima correspond to regions where a transition between layers occurs. In addition, the solvation force curve reveals the presence of a doublet, giving rise to local minima in the two and three layered regimes. This feature has been observed in an earlier GCMC study ${ }^{17}$ where a transition from a two layered bcc to triclinic structures occurred for a fluid confined in a structured slit pore. In both the two and three layered regimes the transformation to a triangular lattice is accompanied by a decrease in the solvation force [Fig. 10(a)]. The sharp drop in the order parameter $\left(\psi_{4}\right)$ in regions corresponding to the local minima in the solvation force signals the transition between square to triangular lattice structures [Fig. 10(a)]. This occurs for both the two and three layered situations. The magnitude of the solvation force at which the square lattice occurs is higher than that of the triangular lattice. Given a fixed number of layers, this decrease is predominantly due to the formation of triangular lattices at larger pore widths. The differences in the magnitude of the solvation force between square to triangular lattices is also seen to decrease with increasing layers. Figure 10(b) where both the order parameters are superimposed reveals that the transition between square to triangular lattices are sharp occurring over a narrow range of pore widths.

\section{E. 10-4 potential}

Before concluding this section we contrast the results obtained with the 10-4-3 fluid-wall potential with that of the 

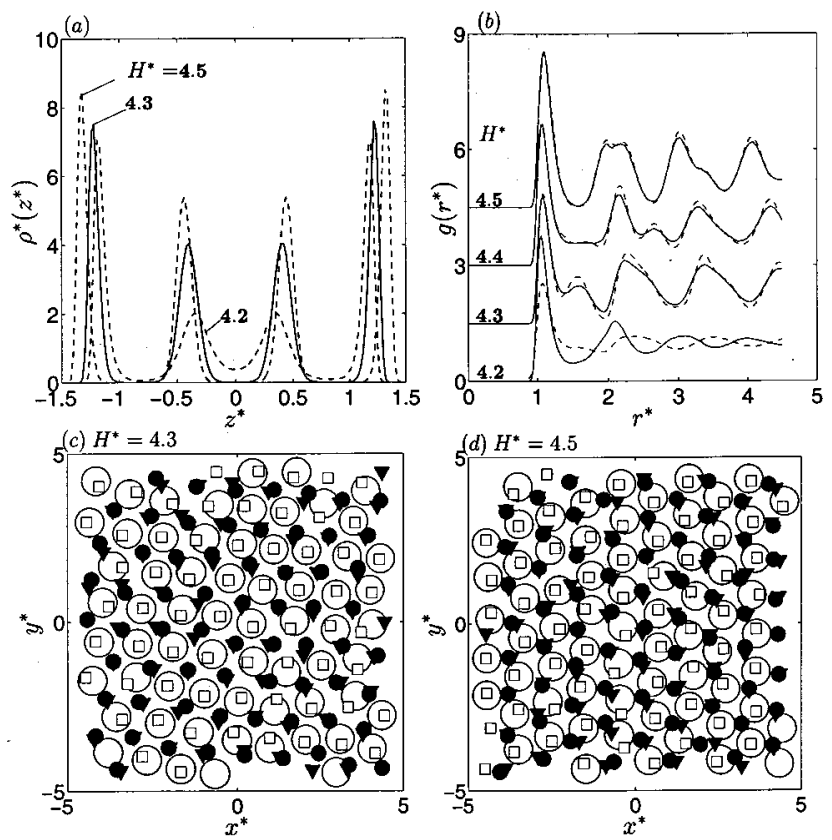

FIG. 9. (a) Density distributions, (b) pair correlation functions, and snapshots (c) and (d) for the four layered regime. The topview snapshots reveal the presence of a square lattice at $H^{*}=4.3$ and a triangular lattice at $H^{*}$ $=4.5$. Both these lattice structures indicate that the stacking is ABAB. The symbols for the snapshots are similar to those used for the three layered snapshots (Fig. 8) with shaded triangles representing the additional layer.

10-4 potential. We note that the GCMC simulations for the 10-4 fluid-wall potential was carried out under identical thermodynamic conditions as the $10-4-3$ potential. Since the overall qualitative features are in general similar ${ }^{46}$ to the results obtained with the 10-4-3 pore only key differences are highlighted. Figure 11 illustrates representative pair correlation functions for pore widths that accommodate 1, 2, and 3 layers. The PCF's in the single layer regime $\left(H^{*}=1.8\right.$ and 2.2) do not show the structure characteristic of a triangular lattice as observed for the 10-4-3 potential [Fig. 3(b)]. In contrast, the structure of the PCF (Fig. 11) reveals a more liquidlike behavior. Although, a small increase in structure is observed at $H^{*}=2.1$ due to increased buckling, the pore fluid still lacks in-plane order. In the two layered regime PCF's indicate the formation of square and triangular lattices at $H^{*}=2.6$ and $H^{*}=2.85$, respectively. Similarly, in the three layered regime, square and triangular lattices are observed at $H^{*}=3.55$ and $H^{*}=3.8$, respectively. In comparison with the 10-4-3 pore the equilibrium pore densities (see legend in Fig. 11) for the 10-4 system are significantly lower. The lower density at smaller pore widths, precludes the formation of an ordered phase in the single layer region. The sequence of square to triangular lattices occur in a qualitatively similar fashion to the 10-4-3 pore, however the overall intensities of the peaks in the PCF's and values of the order parameters (Fig. 11) for the lattices are smaller when compared with those of the 10-4-3 pore, indicating the gradual loss of order due to weaker fluid-wall interactions, and lower pore densities. Further, the square lattices are seen to exist over a smaller range of pore widths and the transition regimes (during which the pore fluid is disordered) between lattice structures occur over a wider range of pore widths
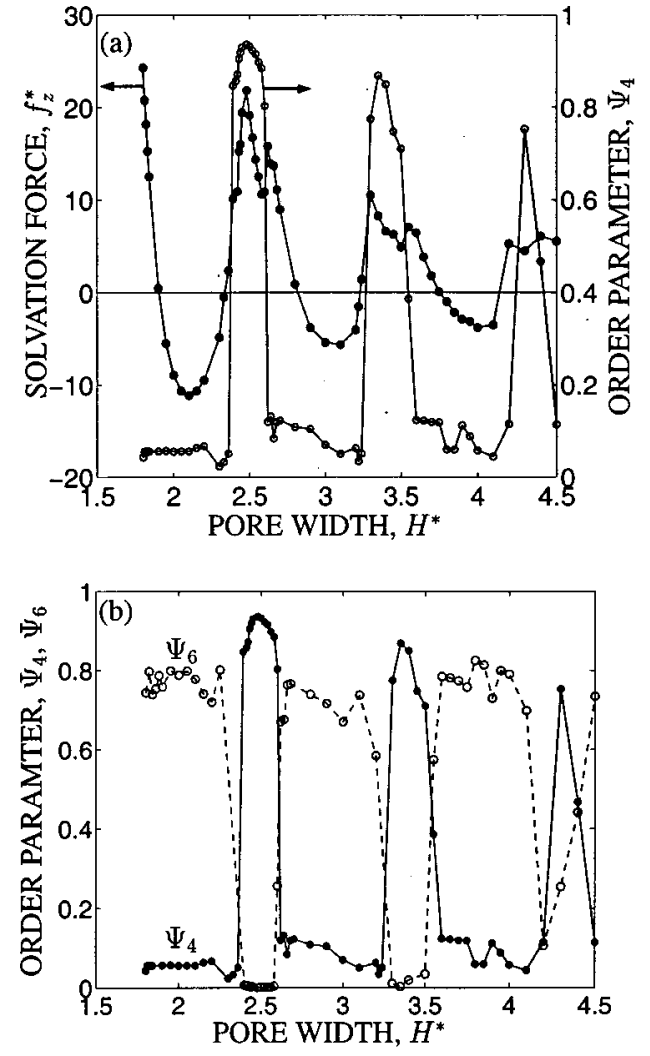

FIG. 10. (a) Solvation force and bond angle order parameter, $\psi_{4}$, as a function of the pore width; 10-4-3 fluid-wall potential. The splitting in the solvation force peaks, in the two and three layered regimes occurs during the transition between square to triangular lattices as seen by the rapid change in $\psi_{4}$ through this region. (b) Order parameters $\psi_{4}$ and $\psi_{6}$ as a function of pore width; 10-4-3 fluid-wall potential. The transformation between square and triangular lattices occurs over a narrow range of pore widths as seen by the sharp changes in the order parameters during the transformation between triangular and square lattices.

when compared with the 10-4-3 pore. Although the solvation force maxima do indicate a weak split (Fig. 11), the variation in the solvation force between the square and triangular lattices is small. Albeit much smaller, when compared with the 10-4-3 pore, the transition between square to triangular lattices is accompanied by a lowering in the solvation force.

\section{DISCUSSION}

Unless otherwise stated we restrict our discussion to the structures observed in the 10-4-3 system. From the density distributions the following regimes, within the resolution of slit widths $\left(\Delta H^{*}=0.02-0.03\right)$ used in our simulations, can be discerned; single layer $1.8 \leqslant H^{*} \leqslant 2.25$; transition between 1 and 2 layers, $2.25<H^{*} \leqslant 2.36$, two layers 2.39 $\leqslant H^{*} \leqslant 3.1$; transition between 2 and 3 layers $3.2 \leqslant H^{*}$ $<3.3$, three layers $3.3 \leqslant H^{*} \leqslant 4.1$ and four layers $4.2 \leqslant H^{*}$ $\leqslant 4.5$. The transition regimes where layering is disrupted, occurs in a small range of pore widths indicating that the confined fluid is in a highly layered state. The series of structures that form are summarized in Fig. 12, where $\mathbf{T}, \mathbf{B}$, and $\mathbf{S}$ denote the triangular, buckled, and square lattice structures, respectively. Figure 12 also illustrates the variation in the number of particles $\langle N\rangle$ with increasing pore widths. Al- 

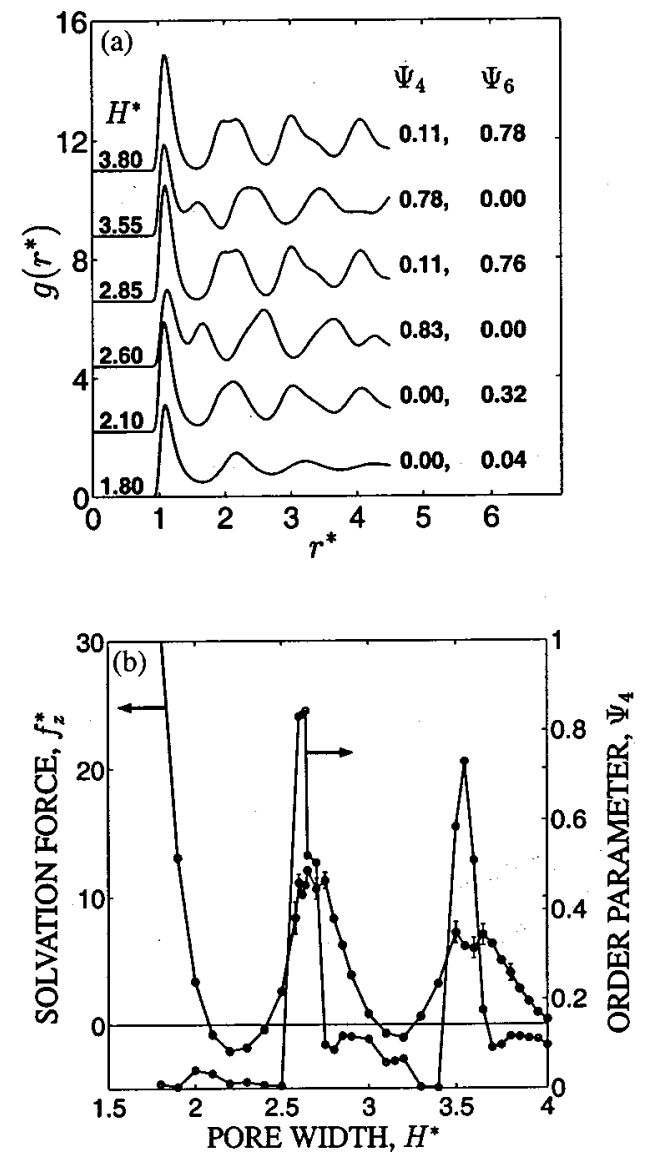

FIG. 11. (a) Pair correlation functions, (b) solvation force and order parameter $\psi_{4}$ as a function of pore width for the 10-4 fluid-wall potential. In general the peaks are broader and order parameters are less, indicating that the structures possess smaller in-plane order when compared with the fluid confined in the 10-4-3 pore. In particular the single layer is liquidlike as indicated by the pair correlation functions at $H^{*}=1.8,2.1$. The pore densities corresponding to $H^{*}=1.8,2.1,2.6,2.85,3.55$, and 3.80 are 0.425 , $0.405,0.543,0.622,0.665$, and 0.700 , respectively.

though simulations in the single layer regime were carried out for a simulation box of length $12 \sigma_{f f}$, for purposes of comparison, the ensemble averaged numbers were scaled to a box of length $9 \sigma_{f f}$. Two dominant jumps in the pore density which occur around $H^{*}=2.4$ and $H^{*}=3.2$ correspond to layering transitions. The transitions from the square to triangular lattices are accompanied by a small increase in pore density as seen in the increasing number of particles as a function of pore width in Fig. 12. These jumps in density are reflected as steps in $\langle N\rangle$. In the two layered regime the two steps correspond to the formation of square and triangular lattices, respectively. In the three layered regime, in addition, we observe a third step which accompanies the transition from hcp to fcc structures.

The in-plane lattice parameters $a^{*}=b^{*}$ represent the lattice parameters in the $x-y$ plane and $c^{*}$ represents the lattice parameter in the $z$ direction assuming a complete unit cell of three layers. $a^{*}$ and $b^{*}$ were estimated from the peaks in $g\left(r^{*}\right)$ and $c^{*}$ was computed from the peak positions of the layered density distributions to within an accuracy of $0.02 \sigma_{f f}$. Representative lattice parameters, structures and $c * / a *$ ratios are summarized in Table III. The square lattices

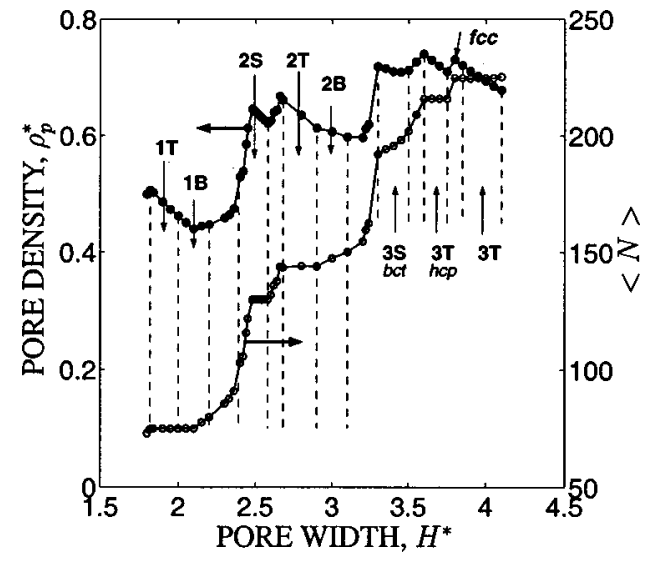

FIG. 12. Reduced pore density, $\rho_{p}^{*}$ vs $H^{*}$ denoting the various phases observed for the 10-4-3 fluid-wall potential. T, triangular; $\mathbf{B}$, buckled; $\mathbf{S}$, square with the numerical prefix indicating the number of layers. The regions where the structures are not marked indicate transitions between layers or phases. The three layer fcc structure is observed at $H^{*}=3.8$.

that form in the two layered regime correspond to the first two basal planes of a bct lattice and the triangular lattices correspond to the first two basal planes of the hep lattice. At $H^{*}=2.39$ where the first square lattice structures were observed, the lattice is somewhat loosely packed $\left(a^{*}=1.16\right)$ as seen in Table III. This lattice parameter decreases as the pore width is increased beyond $H^{*}=2.42$. The $c^{*} / a^{*}$ ratios for the three layered structures indicates that the three layered square lattices correspond to a complete unit cell of the bct lattice and the three layered triangular lattices correspond to a complete unit cell of the hcp lattice. At $H^{*}=3.8$ the ABA stacking transforms to the ABC stacking of a fcc lattice (Fig. 8). The hcp structures in the three layered regime have $c^{*} / a^{*}$ ratios both smaller and larger than the ideal closed packed value of 1.633 , indicating that the ideal packing exists for $3.6<H^{*}<3.7$. It is interesting to note, for the buckled phases, which occur before the admission of an additional layer (formation of square lattices) in the two and three layered regimes, the $c^{*} / a^{*}$ ratio is near 2 , indicating that these structures exist in a highly expanded state.

Buckling was seen to occur prior to the inclusion of an additional layer. In the absence of a quantitative definition to pinpoint the onset of a buckled phase, we indicate buckling to occur when the out of plane deviations exceeds $0.5 \sigma$. In our system buckling occurs in the region $2.0 \leqslant H^{*} \leqslant 2.25$ in the single layered regime, for $2.9 \leqslant H^{*} \leqslant 3.1$ in the two layered regime and for $3.8<H^{*} \leqslant 4.0$ in the three layered regime. Buckling was also found to increase in-plane order as seen in the small increase in $\psi_{6}$ prior to complete disorder. Similar to observations in colloidal suspensions ${ }^{29,30}$ buckling is most pronounced during the transition from the singlelayer to the two layered regime with reduced buckling as the number of layers is increased. In hard sphere simulations, ${ }^{33,34}$ atoms move out-of-plane in a regular fashion giving rise to zig-zag or linear buckled structures. In our simulations buckling was random with no preferred out-ofplane movement. Unlike hard sphere systems the fluid-wall interaction plays an important role in the single layer regime. In our system (similar to other slit pores) the fluid-wall in- 
TABLE III. Lattice parameters $a^{*}$ (in-plane) and $c^{*}$ (out-of-plane) for different pore widths in the two and three layered regimes.

\begin{tabular}{|c|c|c|c|c|c|}
\hline$H^{*}$ & $a^{*}$ & $c^{*} / 2$ & $c^{*}$ & $c^{*} / a^{*}$ & Structures \\
\hline 2.39 & 1.16 & 0.60 & $\cdots$ & 1.034 & $2 \mathbf{S}$ \\
\hline 2.42 & 1.17 & 0.60 & $\cdots$ & 1.025 & $2 \mathbf{S}$ \\
\hline 2.45 & 1.14 & 0.66 & $\cdots$ & 1.158 & $2 \mathbf{S}$ \\
\hline 2.48 & 1.10 & 0.70 & $\cdots$ & 1.273 & $2 \mathbf{S}$ \\
\hline 2.50 & 1.12 & 0.68 & $\cdots$ & 1.214 & $2 \mathbf{S}$ \\
\hline 2.54 & 1.09 & 0.72 & $\cdots$ & 1.321 & $2 \mathbf{S}$ \\
\hline 2.66 & 1.11 & 0.84 & $\cdots$ & 1.514 & $2 \mathbf{T}$ \\
\hline 2.68 & 1.11 & 0.86 & $\cdots$ & 1.550 & $2 \mathbf{T}$ \\
\hline 2.70 & 1.10 & 0.88 & $\cdots$ & 1.60 & $2 \mathbf{T}$ \\
\hline 2.80 & 1.10 & 0.94 & $\cdots$ & 1.709 & $2 \mathbf{T}$ \\
\hline 2.90 & 1.10 & 1.00 & $\cdots$ & 1.818 & $2 \mathbf{T B}$ \\
\hline 3.00 & 1.10 & 1.06 & $\cdots$ & 1.927 & $2 \mathrm{~TB}$ \\
\hline 3.10 & 1.10 & 1.08 & $\cdots$ & 1.964 & $2 \mathrm{~TB}$ \\
\hline 3.30 & 1.08 & $\cdots$ & 1.48 & 1.370 & $3 \mathbf{S}$ \\
\hline 3.40 & 1.08 & $\cdots$ & 1.58 & 1.463 & $3 \mathbf{S}$ \\
\hline 3.50 & 1.08 & $\cdots$ & 1.64 & 1.490 & $3 \mathbf{S}$ \\
\hline 3.60 & 1.10 & $\cdots$ & 1.74 & 1.582 & 3T-hcp \\
\hline 3.70 & 1.10 & $\cdots$ & 1.84 & 1.673 & 3T-hcp \\
\hline 3.80 & 1.10 & $\cdots$ & 1.94 & 1.764 & $3 \mathbf{T}-\mathrm{fcc}$ \\
\hline 3.90 & 1.10 & $\cdots$ & 2.00 & 1.818 & $3 \mathbf{T}$ \\
\hline 4.00 & 1.08 & $\cdots$ & 2.10 & 1.944 & $3 \mathbf{T}$ \\
\hline 4.05 & 1.08 & $\cdots$ & 2.14 & 1.981 & $3 \mathbf{T}$ \\
\hline 4.10 & 1.08 & $\cdots$ & 2.22 & 2.056 & $3 \mathbf{T}$ \\
\hline
\end{tabular}

teraction potential has a minimum at the center of the pore for $1.8 \leqslant H^{*} \leqslant 2.1$. As the pore width is increased the minimum from the pore center shifts toward the walls giving rise to the two layered regime. During this transition which occurs for $2.1<H^{*} \leqslant 2.25$, the fluid-wall interaction potential shows a broad weak minima. It is during this stage that buckling is most pronounced. This trend is also clearly reflected in the broadening of the density distributions shown in Fig. 3(a). The transition from one to two layers is dominated by strong fluid-wall interactions (greatest at smaller pore widths) which probably offsets the energetic gain due to ordered buckling. A preliminary Voronoi analysis of defects indicate that the defects in the single layer buckled phase was the greatest when compared with the buckled structures at greater pore widths.

During the transition from triangular to square lattices in the two and three layered regimes, buckling in the wall layers are less pronounced when compared with the single layer regime since the particles are located in the fluid-wall potential energy minima. However, snapshots of the particles indicate a deviation from regular $\mathrm{AB}$ stacking in the two layered buckled regime [Fig. 6(d)]. This phenomenon akin to a relative shearing of layers has also been observed in colloidal suspensions. ${ }^{30}$ In the three layered regime this shearing effect [Fig. 8(d)] gives rise to structures that have a layering between ideal $\mathrm{ABC}$ and $\mathrm{ABA}$ stacking in the regime $3.8<H^{*} \leqslant 4$.1. Although hard sphere packing ${ }^{29}$ (in the absence of fluid-fluid interactions) do indicate that both $\mathrm{ABA}$ and $\mathrm{ABC}$ stackings are equally preferred, we are unable to explain the preference toward the $\mathrm{ABC}$ stacking at $H^{*}$ $=3.8$.

We point out that the rhombic phase, which has been previously observed in hard sphere simulations ${ }^{33}$ during the transformation between two layered square to triangular lat- tices was not observed in this study. We looked for the possible occurrence of these structures by carrying out simulations in this regime $\left(2.58<H^{*}<2.62\right)$ with a fine resolution in the pore width. However examination of the snapshots and the PCF's did not reveal the presence of this structure. Perhaps a more sensitive order parameter ${ }^{33,34}$ is required to make a more definitive statement on the existence of this phase.

Since the thermodynamic state of the bulk fluid determines the density of the pore fluid and consequently its state of order, it is expected to have a strong influence in determining the presence of these transitions and the extent to which these transitions would persist as the pore width is increased. Simulations carried out with a lower density bulk fluid $\left(T^{*}=1.2\right.$ and $\left.\rho^{*}=0.661\right)$ did not reveal any in-plane order in the $10-4$ pore. ${ }^{47}$ For a given fluid, a complete mapping of the phase diagram would depend on both the thermodynamic state of the bulk fluid and fluid-wall interaction strength.

Lastly we mention that our findings are consistent with previous simulation studies of methane on graphite where the strong fluid-solid interaction for this system leads to an increase in the freezing temperature relative to the bulk. In the global phase diagram developed for freezing in nanopores an elevation and depression in the freezing temperature relative to the bulk was related to a parameter $\alpha$ which depends on the fluid-wall interaction strength. ${ }^{48}$ This study reveals that an elevaton in freezing temperature should occur for $\alpha \geqslant 1.15$. In agreement with these finding we observe an elevation in the freezing temperature for the 10-4-3 pore where $\alpha=2.16$. However for the 10-4 system although the value of $\alpha=1$ is slightly below 1.15 , we still see a frozen phase though less structured than the 10-4-3 pore.

\section{SUMMARY AND CONCLUSIONS}

GCMC simulations have been used to study the structure of a fluid confined in a smooth slit pore. Unlike previous studies we choose a bulk state point lying close to the liquid-solid freezing line. This not only results in solidification of the pore fluid but a sequence of solid phases are observed with increasing pore width. In the single layer regime the fluid forms a triangular lattice in the 10-4-3 pore due to the increased pore densities. For the two and three layered structures, the fluid first forms a square lattice which transforms into a triangular lattice with increasing pore width and pore fluid density. Buckled phases which occur during the transformation from $n$ to $n+1$ layers is a result of structures maintaining their in-plane triangular lattice structure with increased out-of-plane disorder. The observed structures are classified based on the 3D unit cells. The square lattices are bct in structure and the triangular lattices are hcp. In the three layered regime an additional transformation occurs due to a change in the stacking from ABA (hcp) at lower pore widths to ABC (fcc) at larger pore widths. The study reveals, that hcp structures possessing both positive and negative deviations from an ideal lattice can exist under confinement. In addition stable triangular lattices can exist under a highly expanded state. 
Since the sequence of transitions observed in molecularly thin films, such as the ones studied here, are qualitatively similar to solid-solid transformations in colloidal systems we compare the structures with those observed in confined colloidal suspensions. Unlike hard sphere systems, the nature of the wall can influence the density and therein the structure of the confined fluid. Simulations with a weakly attractive 10-4 fluid-wall potential reveals these differences. Although the single layer structure is disordered due to the lower pore densities, the sequence of transitions is qualitatively similar to that observed in a 10-4-3 pore.

Gas to liquid and more recently liquid to solid transitions under molecular confinement has been the subject of extensive investigations using molecular simulations. However, the formation of frozen phases and transitions between them, such as the ones reported in this study have been largely unexplored. Although, our study has been limited to simple spherical molecules, it suggests that more complex frozen phases can exist in confined molecular systems. Recent surface force experiments ${ }^{49,50}$ on water confined to molecular layers indicate the subnanometer confined films are in a high state of order. Although our study is restricted to determining the presence of a solid phase by computing structural quantities, a complete thermodynamic treatment would involve computing the free energies of the various phases.

${ }^{1}$ J. N. Israelachvili, Intermolecular and Surface Forces: With Applications to Colloidal and Bioligical Systems (Academic, London, 1985).

${ }^{2}$ J. Klein and E. Kumacheva, J. Chem. Phys. 108, 6996 (1998).

${ }^{3}$ M. L. Gee, P. M. McGuiggan, and J. N. Israelachvili, J. Chem. Phys. 93, 1895 (1990).

${ }^{4}$ L. A. Demirel and S. Granick, Phys. Rev. Lett. 77, 2261 (1996).

${ }^{5}$ L. A. Demirel and S. Granick, J. Chem. Phys. 115, 1498 (2001).

${ }^{6}$ M. Heuberger, M. Zäch, and N. D. Spencer, Science 292, 905 (2001).

${ }^{7}$ I. Snook and W. van Megen, J. Chem. Phys. 72, 2907 (1980).

${ }^{8}$ M. Schoen, D. J. Diestler, and J. H. Cushman, J. Chem. Phys. 87, 5464 (1987).

${ }^{9}$ J. Gao, W. D. Luedtke, and U. Landmann, J. Phys. Chem. B 101, 4013 (1997).

${ }^{10}$ H. T. Davis, Statistical Mechanics of Phases, Interfaces, and Thin Films (VCH, New York, 1995).

${ }^{11}$ C. L. Rhykerd, M. Schoen, D. J. Diestler, and J. H. Cushman, Nature (London) 330, 461 (1987).

${ }^{12}$ P. A. Thompson and M. O. Robbins, Phys. Rev. A 41, 6830 (1990).
${ }^{13}$ S. A. Somers and H. T. Davis, J. Chem. Phys. 96, 5389 (1992).

${ }^{14}$ S. A. Somers, K. G. Ayappa, A. V. McCormick, and H. T. Davis, Adsorption 2, 33 (1996).

${ }^{15}$ M. Miyahara and K. E. Gubbins, J. Chem. Phys. 106, 2865 (1997).

${ }^{16}$ C. Ghatak and K. G. Ayappa, Phys. Rev. E 64, 051507 (2001).

${ }^{17}$ P. Bordarier, B. Rousseau, and A. H. Fuchs, Mol. Simul. 17, 199 (1996).

${ }^{18}$ L. D. Gelb, K. E. Gubbins, R. Radhakrishan, and M. SliwinskaBartkowiak, Rep. Prog. Phys. 62, 1573 (1999).

${ }^{19}$ R. Radhakrishanan, K. E. Gubbins, A. Watanabe, and K. Kaneko, J. Chem. Phys. 111, 9058 (1999).

${ }^{20}$ R. Radhakrishanan, K. E. Gubbins, and M. Sliwinska-Bartkowiak, J. Chem. Phys. 112, 11048 (2000).

${ }^{21}$ H. Dominguez, M. P. Allen, and R. Evans, Mol. Phys. 96, 209 (1999).

${ }^{22}$ K. Morishige and K. Kawano, J. Chem. Phys. 110, 4867 (1999).

${ }^{23}$ K. Koga, H. Tanaka, and X. C. Zeng, Nature (London) 408, 564 (2000).

${ }^{24}$ J. Slovak, K. Koga, and H. Tanaka, Phys. Rev. E 60, 5833 (1999).

${ }^{25}$ J. Kamakshi and K. G. Ayappa, Langmuir 17, 5245 (2001).

${ }^{26}$ D. W. Brown, P. E. Sokol, and S. N. Ehrlich, Phys. Rev. Lett. 81, 1019 (1998).

${ }^{27}$ K. Morhishige and Y. Ogisu, J. Chem. Phys. 114, 7166 (2001).

${ }^{28}$ D. Wallacher and K. Knorr, Phys. Rev. B 63, 104202 (2001).

${ }^{29}$ P. Pieranski, L. Strzelecki, and B. Pansu, Phys. Rev. Lett. 50, 900 (1983).

${ }^{30}$ D. H. van Winkle and C. A. Murray, Phys. Rev. A 34, 562 (1986).

${ }^{31}$ C. A. Murray, W. O. Sprenger, and R. A. Wenk, Phys. Rev. B 42, 688 (1990).

${ }^{32}$ J. E. Hug, F. van Swol, and C. F. Zukoski, Langmuir 11, 111 (1995).

${ }^{33}$ M. Schmidt and H. Löwen, Phys. Rev. Lett. 76, 4552 (1996).

${ }^{34}$ M. Schmidt and H. Löwen, Phys. Rev. E 55, 7228 (1997).

${ }^{35}$ R. Zangi and S. A. Rice, Phys. Rev. E 61, 660 (2000).

${ }^{36}$ K. J. Strandburg, Rev. Mod. Phys. 60, 161 (1988).

${ }^{37}$ A. H. Marcus and S. A. Rice, Phys. Rev. E 55, 637 (1996).

${ }^{38}$ S. Neser, C. Bechinger, P. Leiderer, and T. Palberg, Phys. Rev. Lett. 79, 2348 (1997)

${ }^{39}$ A. Bonissent, P. Pieranski, and P. Pieranski, Philos. Mag. A 50, 57 (1984).

${ }^{40}$ W. A. Steele, The Interaction of Gases with Solid Surfaces (Pergamon, Oxford, 1974).

${ }^{41}$ R. Agarwal and D. A. Kofke, Mol. Phys. 85, 43 (1995).

${ }^{42}$ A. Lofti, J. Vrabec, and J. Fischer, Mol. Phys. 76, 1319 (1992).

${ }^{43}$ W. van Megen and I. Snook, J. Chem. Phys. 74, 1409 (1981).

${ }^{44}$ K. J. Johnson, J. A. Zollweg, and K. E. Gubbins, Mol. Phys. 78, 591 (1993).

${ }^{45}$ M. P. Allen and D. J. Tildesley, Computer Simulation of Liquids (Clarendon, Oxford, 1987).

${ }^{46}$ C. Ghatak and K. G. Ayappa, Colloids Surf. A 205, 111 (2002).

${ }^{47}$ C. Ghatak, Master's thesis, Indian Institute of Science, 2000.

${ }^{48}$ R. Radhakrishanan, K. E. Gubbins, and M. Sliwinska-Bartkowiak, J. Chem. Phys. 116, 1147 (2002).

${ }^{49}$ U. Raviv, P. Laurat, and J. Klein, Nature (London) 413, 51 (2001).

${ }^{50}$ Y. Zhu and S. Granick, Phys. Rev. Lett. 87, 096104 (2001). 